\title{
Postpartum Early Rupture Interstitial Ectopic Pregnancy: A Life Threatened Event Due to Delayed Diagnosis. (Case Report)
}

\author{
Dr. Muad Gamil M Haidar ${ }^{1 *}$, Nuha Ahmed H. Sharaf ${ }^{2}$
}

\begin{abstract}
${ }^{1}$ Department of General Surgery and Endoscopy, AlGamhoria Teaching Hospital, University of Aden, Faculty of Medicine and Health Sciences, and Head of General Surgery at Al Naqeeb Hospital, Aden, Yemen

${ }^{2}$ Department of Surgery, University of Aden, Faculty of Medicine and Health Sciences, and General Surgeon at Al Naqeeb Hospital, Adan, Yemen
\end{abstract}

DOI: $10.36347 /$ sjmcr.2021.v09i04.006

| Received: 03.03.2021 | Accepted: 02.04.2021 | Published: 09.04.2021

*Corresponding author: Muad Gamil M Haidar

Abstract

Case Report

Background: Ectopic pregnancy (EP) is a common and potentially life- and fertility-threatening condition in early pregnancy. The majority of EP are tubal. Interstitial EP is a rare site of implantation and usually associated with the highest risk of massive and uncontrollable bleeding. Despite the accurate and different diagnostic methods, the correct diagnosis of interstitial EP considered very difficult in early stages. Case Presentation: 20- year old fully lactating female gravida 2 para 1 with a history of normal vaginal delivery and amenorrhea for 5 months, presented with sudden acute abdominal pain 2 days prior to admission. On examination she had pallor tachycardic febrile and hypotensive. Her abdomen was distended and tender. She had a $\beta$-human chorionic gonadotropin level of $70 \mu \mathrm{IU} / \mathrm{ml}$. The abdominal ultrasound showed only a moderate intra-abdominal collection. Emergency laparoscopic intervention revealed a large hemoperitoneum, and bleeding from a ruptured left interstitial ectopic pregnancy. The procedure was accomplished by hand-assisted suturing of the defect to control the bleeding. The patient was discharged after two days in good condition. Discussion: Early rupture of interstitial ectopic pregnancy is rare and seldom reported. Its diagnosis and management are challenging and requires prompt intervention. Although the diagnosis of ectopic pregnancy was valued in our evaluation, the exact diagnosis was unclear because the patient came in an acute abdomen, the fear of viscus perforation was existed, and the possibility of getting pregnancy in postpartum period was unexpected. Conclusion: Ruptured interstitial EP is a life threatened entity. The diagnosis of early ruptured interstitial EP is challenging in an emergency sitting. The final diagnosis usually confirmed during laparoscopic or surgical intervention.

Keywords: Ectopic pregnancy Ruptured ectopic pregnancy, Interstitial ectopic pregnancy, management of ectopic pregnancy, laparoscopic role in gynecological emergencies, lactational amenorrhea.

Abbreviations: Ectopic Pregnancy (EP), Interstitial Ectopic Pregnancy (ISEP), Ultrasound (US), Beta Chorionic Gonadotropin ( $\beta$-hCG).

Copyright $\odot 2021$ The Author(s): This is an open-access article distributed under the terms of the Creative Commons Attribution 4.0 International License (CC BY-NC 4.0) which permits unrestricted use, distribution, and reproduction in any medium for non-commercial use provided the original author and source are credited.

\section{INTRODUCTION}

Ectopic pregnancy is the implantation of a fertilized ovum outside the uterine cavity and it is thought to affect $1-2 \%$ of pregnancies. It represents the most common cause of pregnancy-related mortality in the first trimester $(9-14 \%$ mortality rate) $[1,2]$. The majority of ectopic $(93-97 \%)$ pregnancies are located within the fallopian tube $[1,3]$.

Interstitial pregnancy occurs in the interstitial segment of the fallopian tube (lateral to the round ligament) that lies within the muscular wall of the uterus and accounts for up to $1-3 \%$ of all ectopic pregnancies $[3,4]$.

Nevertheless, ruptured EP continues to occur, often because the clinician or the patient did not recognize the early signs and symptoms of the condition [5].

Ruptured ISEP can result in catastrophic hemorrhage with massive blood loss from the vascular anastomosis between the uterine and the ovarian arteries (Sampson's artery) and the mortality rate is 6-7 
times higher than that in classical ectopic pregnancy [6].

Here we introduce a considered very rare case of a life threatened early ruptured ISEP in a young Arab/Yemeni lady diagnosed during a laparoscopic intervention. Her ectopic pregnancy was unexpected and unusual because the patient had a history of normal vaginal delivery 5 months earlier, and she had been in a period of lactational amenorrhea. Up to the best of our knowledge the ISEP in such condition had not been described in the literatures review.

\section{Case Presentation}

A 20-years-old Yemeni lady, gravida 2 para 1, with a history of normal vaginal delivery 5 months ago, presented at one-month gestation (according to the patient's discerption) with sudden general and progressive abdominal pain commencing 2 days prior to presentation. She denied any vaginal bleeding or leaked liquor. The pain was associated with fever, nausea, vomiting, and dizziness. She also provided a history of amenorrhea and full breastfeeding since her delivery.

The patient was hemodynamically unstable. She was pallor, febrile, and slightly dyspneic, with blood pressure of $90 / 60 \mathrm{~mm} / \mathrm{Hg}$, pulse rate 110 beats $/ \mathrm{m}$ (weak and thready pulse), and respiratory rate 18 breath $/ \mathrm{m}$ with normal breathing sounds.

The abdomen was symmetrically distended, and a suprapubic transverse old surgical skin scar was visible. Figure 1 there were scant bowel sounds detected by auscultation. The abdomen was diffusely tender with positive rebound tenderness. The digital rectal examination showed an empty and tender rectum.

The patient's past medical history includes an unreported abdominal operation for left ovarian cyst two years ago. Blood laboratory investigation showed, HB 5mg/dl, WBC 12000, $\beta$-hCG $70 \mu \mathrm{IU} / \mathrm{ml}$, blood urea $40 \mathrm{mg} / \mathrm{dl}$, and creatinine $1.8 \mathrm{mg} / \mathrm{dl}$.

Bedside urgent abdominal US revealed moderate intra-abdominal free fluid collection, no intrauterine pregnancy, and no other pathologies were detected.

The patient immediately resuscitated by blood and fluids transfusion. Intravenous antibiotics analgesics and proton pump inhibitor was given too. Naso-gastric tube and urine catheter had put in place.

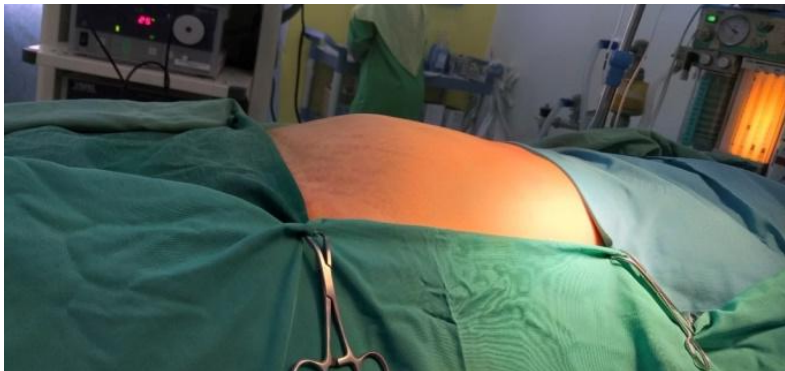

Fig-1: Pre-operative image showing the abdominal distention (Red arrow), and the old suprapubic scar of the previous operation of the patient (White arrow)

So, the decision was taken for urgent laparoscopic intervention. During the procedure: there was a large amount of intra-abdominal blood collection $>1500 \mathrm{ml}$. Figure 2 Blood was evacuated using suction with inspection of pelvic organs.

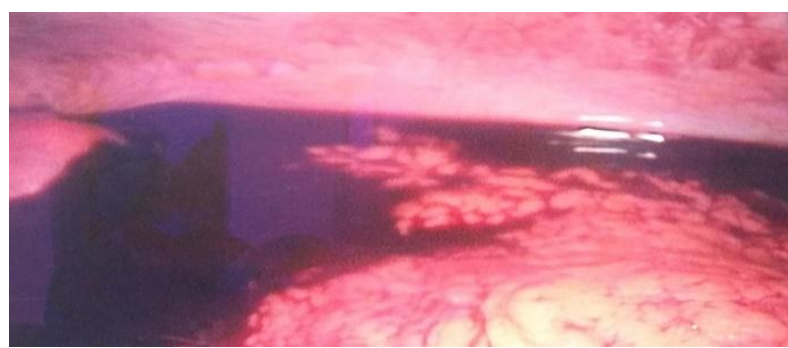

Fig-2: The initial laparoscopic image showing large intraabdominal blood collection

There was a free dark soft tissue material (the embryo) measuring about $7 \times 5 \mathrm{~cm}$ floated in the pelvis. An insidious blood Hemorrhage was coming from a defect at the junction of the left fallopian tube to the uterus indicated a ruptured left ISEP. Figures 3, 4

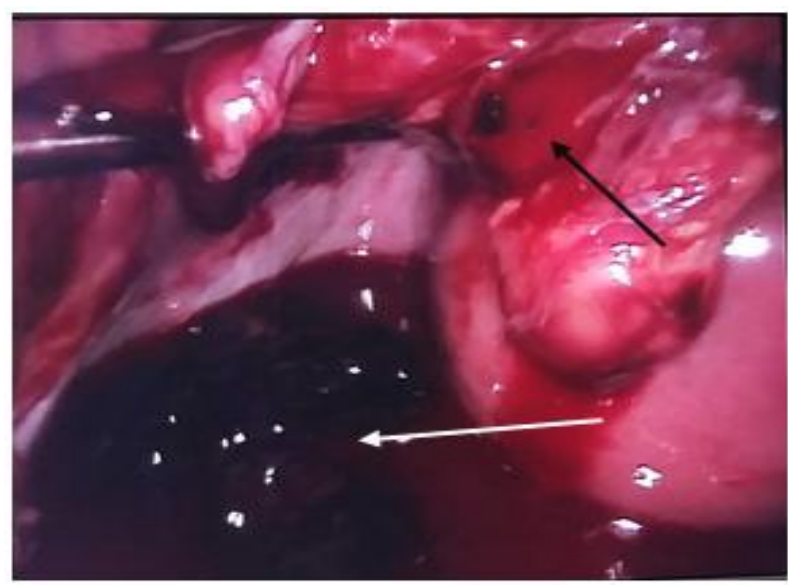

Fig-3: Insidious blood ooze from the rupture uterine corn defect (Black arrow). The floated embryo (White arrow) 


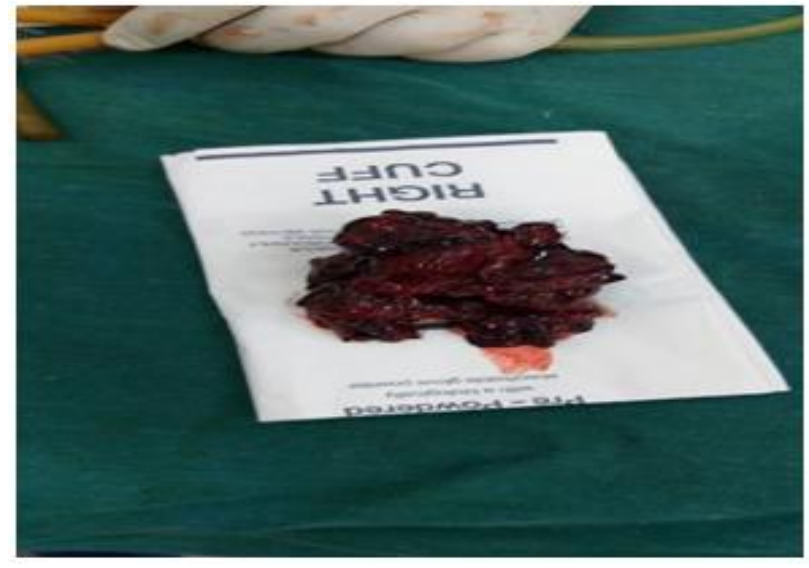

Fig-4: The embryo after extraction

It was difficult to control the blood ooze laparoscopically due to the friability of the implantation site. The procedure had progressed to open suprapubic small transverse incision to deliver out the uterus. Figure 5 A direct two layers' hand sewing of the uterine horn defect was done to control the bleeding.

The patient had an uneventful postoperative course and discharged after 2 days in good condition.

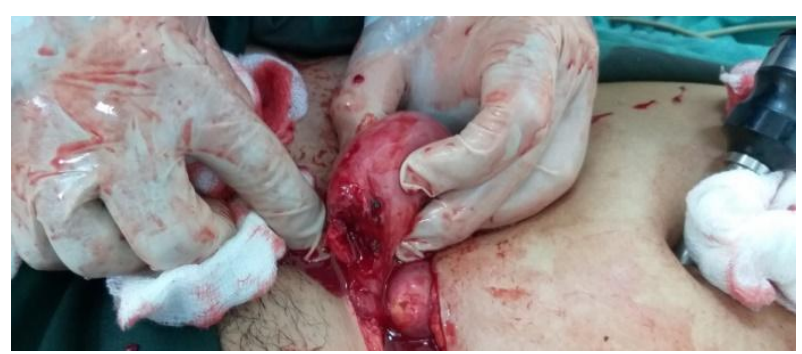

Fig-5: The extracted uterus, and the site of ruptured site of the ISEP

\section{DISCUSSION}

Ectopic pregnancy at any location is a serious problem. Although great advances in the diagnostic and therapeutic management of EP have been made over the last two decades, misdiagnosis and delayed intervention remain the leading clinical causes of maternal death. Therefore, early detection is crucial to reduce morbidity and mortality $[3,6,7]$.

As in other type of EP, the predisposing factors for ISEP may include pelvic inflammatory disease, previous ipsilateral or bilateral salpingectomy, previous ectopic and pelvic surgery, tumors, uterine anomalies and in vitro fertilization [2, 4, 6]. However, half of all women who receive a diagnosis of an EP do not have any known risk factors [8]. The only risk factor we obtained in this case was a history of previous ipsilateral salpingectomy that had done before two years.

The general clinical manifestations of EP are nonspecific. Non ruptured ISEP can be presented with the classic symptoms of EP namely, amenorrhea, vaginal bleeding, and abdominal pain. It can be diagnosed rapidly and accurately with the use of transvaginal ultrasonography in conjunction with a quantitative serum $\beta$-hCG test $[8,9]$.

While all ectopic pregnancies are associated with a risk of hemorrhage, ISEP are associated with the highest risk of massive, uncontrollable bleeding. In contrast to the common clinical notion that rupture occurs only between 12 and 16 weeks, in interstitial pregnancies rupture could happen at any time in early pregnancy as it was happened in this case [10].

Imaging studies (CT and MRI) should be useful for those patients that are hemodynamically and clinically stable [2]. Managing an ISEP is dependent upon whether the ectopic pregnancy has ruptured and the stability of the patient [10].

The safety and effectiveness that laparoscopic procedures offer in gynecological surgeries make them the preferred methods for treating a great variety of gynecologic pathologies $[11,14,15]$.

In female patients in childbearing age with signs and symptoms of acute abdomen, close attention should be paid to her menstrual and gyn-obstetric history and EP should be excluded. In general, patients with resuscitatable acute abdomen as in our case, laparoscopic intervention has the advantages as a minimally invasive procedure, it can make accurate diagnosis and pointing to the underlining causes, in addition to resolving many manageable intra-abdominal acute surgical issues depending on the experience of the surgeon and the availability of the required instruments. Its outcome is outstanding in decreasing the high morbidity of a big midline laparotomy.

In the past, laparotomy with either hysterectomy or cornual resection was advocated in ISEP, [4] although still, radical surgery is necessary in cases where hemorrhage is life-threatening. [6]

Breastfeeding can be up to $98 \%$ effective in preventing pregnancy for the first six months' postpartum as long as the woman remains amenorrhoeic and is fully or nearly fully breastfeeding [16]. This patient had in lactational amenorrhea for 4 months after her recent delivery and achieving pregnancy in this period being unexpected and unusual.

This case had presented in acute abdomen. Although we had some concern about the possible perforated peptic ulcer or perforated acute appendicitis, our evaluation was more confined to a ruptured EP. As the patient's condition was critical, we could not spend more time in evaluating the case by serial $\beta$ - hCG follow up, and we could not send the patient for further imaging studies. The Gyno-obstetric surgeon was not 
reachable at that time for additional evaluation. After adequate resuscitation, the final diagnosis of ISEP was made during laparoscopic intervention.

This reported case could be supporting the importance of awareness and high clinical suspicion for such a life-threatening condition to avoid maternal morbidity and mortality.

\section{Conclusion}

Ruptured interstitial ectopic pregnancy is a rare and a life threatened entity, and its diagnosis considered challenging. The positive $\beta$-hCG, and the negative intrauterine fetus with the intraabdominal collection detected by US raise the index of suspicion of a ruptured EP, however, the final diagnosis of early ruptured ISEP usually made intraoperatively by means of laparoscopic or open surgical intervention. Hemodynamically unstable cases need for more prompt intervention without delay rather than diagnostic evaluation.

\section{DECLARATION}

Ethical approval and consent to participate:

Not applicable.

\section{Consent for publication}

Written informed consent was obtained from the patient for publication of this case report and accompanying images. A copy of the written consent is available for review by the Editor-in-Chief of this journal.

\section{Availability of data and material \\ Available upon request.}

\section{Competing interests}

The authors declare that they have no competing interests.

\section{Funding}

No funding was received to conduct this article.

\section{Authors' contributions}

$\mathrm{MH}$, the first surgeon for this reported patient, researched the literature review, contributed to the discussion, conclusion, and photographs, and did the final manuscript format. NS researched the literature review, contributed to the manuscript revision, researched the archived files to obtain the patient information and direct contact with patient and relatives, and contributed to the list of references.

\section{REFERENCES}

1. Alagbe OA, Adeniyi TO, Abayomi OA, Onifade EO. Interstitial ectopic pregnancy: a case report. Pan African Medical Journal. 2017;28(1):132.

2. Santa LG, Retortillo J An P, Durán EV, Liza LN, Ruptured ectopic pregnancy, Eurorad. 2015, avialable at https://www.eurorad.org/case/12809 , DOI:10.1594/EURORAD/CASE.12809

3. Badr S, Ghareep AN, Abdulla LM, Hassanein R. Ectopic pregnancy in uncommon implantation sites. The Egyptian Journal of Radiology and Nuclear Medicine. 2013, 44(1), 121-130.

4. Yassin AS, Taha MS. Interstitial Ectopic Pregnancy, Diagnosis and Management: A Case Report and Literature Review. Ann Clin Case Rep. 2017; 2: 1352

5. Ectopic pregnancy - United States, 1990-1992. MMWR Morb Mortal Wkly Rep 1995; 44:46-8. Centers for Disease Control and Prevention (CDC). Ectopic pregnancy--United States, 1990-1992. MMWR Morb Mortal Wkly Rep. 1995;44(3):4648.

6. Di Tizio L, Spina MR, Gustapane S, D'Antonio F, Liberati M, Interstitial pregnancy: From medical to surgical approach-report of three cases. Case reports in obstetrics and gynecology, 2018.2018.

7. Lu Q, Wang Y, Sun X, Li Y, Wang J, Zhou Y, Wang $Y$. the diagnostic role of the $\beta$-hcG discriminatory zone combined with the endometrial pattern for ectopic pregnancy in chinese women. Scientific reports. 2019 Sep 24;9(1):1-7.

8. Barnhart KT. Ectopic pregnancy. New England Journal of Medicine. 2009 Jul 23;361(4):379-87.

9. Murray $\mathrm{H}$, Baakdah $\mathrm{H}$, Bardell $\mathrm{T}$, Tulandi $\mathrm{T}$. Diagnosis and treatment of ectopic pregnancy. Cmaj. 2005 Oct 11;173(8):905-12.

10. Rizk B, Holliday CP, Abuzeid M. Challenges in the diagnosis and management of interstitial and cornual ectopic pregnancies. Middle East Fertility Society Journal. 2013 Dec 1;18(4):235-40.

11. Aulestia SN, Cantele H, Leyba JL, Navarrete M, Llopla SN. Laparoscopic diagnosis and treatment in gynecologic emergencies. JSLS: Journal of the Society of Laparoendoscopic Surgeons. 2003 Jul;7(3):239.

12. Wood C, Maher P. The place of laparoscopic surgery in surgical gynaecology. Aust N Z J Obstet Gynaecol. 1998; 38:440-44

13. Diaz D, Barron J, Rojas G, Kably A. Gynecologic laparoscopy at the ABC Hospital. Analysis of 882 cases. Ginecol Obstet Mex. 1998; 66:395-398

14. Ramos R, Kennedy KI, Visness CM. Effectiveness of lactational amenorrhoea in prevention of pregnancy in Manila, the Philippines: noncomparative prospective trial. BMJ. 1996 Oct 12;313(7062):909-12. 